Published in Fluct. Noise Lett. 1, L27-33 (2001).

\title{
NOISE-INDUCED ESCAPE FROM THE LORENZ ATTRACTOR
}

\author{
V.S. ANISHCHENKO, I.A. KHOVANOV, N.A. KHOVANOVA \\ Physics Department, Saratov State University, Astrakhanskaya str. 83, \\ 410026 Saratov, Russia \\ wadim@chaos.ssu.runnet.ru,igor@chaos.ssu.runnet.ru, \\ khovanova@chaos.ssu.runnet.ru \\ D.G. LUCHINSKY, P.V.E. McCLINTOCK \\ Department of Physics, Lancaster University, Lancaster, LA1 $4 Y B$, UK \\ d.luchinsky@lancaster.ac.uk,p.v.e.mcclintock@lancaster.ac.uk \\ Received (received date) \\ Revised (revised date) \\ Accepted (accepted date)
}

\begin{abstract}
Noise-induced escape from a quasi-hyperbolic attractor in the Lorenz system is investigated via an analysis of the distributions of both the escape trajectories and the corresponding realizations of the random force. It is shown that a unique escape path exists, and that it consists of three parts with noise playing a different role in each. It is found that the mechanism of the escape from a quasi-hyperbolic attractor differs from that of escape from a non-hyperbolic attractor. The possibility of calculating the escape probability is discussed.
\end{abstract}

Keywords: Noise-induced escape, quasi-hyperbolic attractor, non-equilibrium fluctuations, prehistory probability distribution

\section{Introduction}

One of the longstanding unsolved problems in the theory of fluctuations is that of noise-induced escape from a chaotic attractor [1-3]. Chaotic systems are widespread in nature, and the study of their dynamics in the presence of noise is a topic of broad interdisciplinary interest whose potential applications include e.g. stabilization of the voltage standard [4] and laser systems [5], neuron dynamics [6], macromolecular transport in biological cells [7], and the control of migration in multistable systems [8-10].

The difficulty in solving the fluctuational escape problem stems largely from the fact that systems possessing strange attractors are far from thermal equilibrium: no general methods are available for estimation of the escape probabilities in such 
systems. Furthermore, the application of asymptotic methods, e.g. WKB-like approximations [11] for solution of the Fokker-Planck equation, requires the definition of the generally unknown boundary conditions and relies on the existence of the so-called most probable escape path which needs to be justified in each particular case.

It has recently been shown, however, that fluctuational dynamics can be investigated directly through measurements of the so-called prehistory probability distribution [12-15], so that it has become possible to use analogue and digital methods to examine situations where the use of analytic methods still remains problematic. In this paper we apply this technique to the investigation of noise-induced escape from a quasi-hyperbolic attractor.

The essence of the prehistory technique lies in the idea that, when the system moves to a given remote state, it does so along an optimal path under the action of a large fluctuation [16]. The dynamical variables of the system and of the fluctuational force are recorded simultaneously, and the corresponding prehistory distributions are built $[12-15]$. This allows one to define the optimal escape path, and the corresponding optimal fluctuational force as maxima of prehistory distributions. With a knowledge of the escape path and the corresponding force, we can hope to calculate the escape probability in the Lorenz system analytically and to develop ways of controlling (and perhaps exploiting) large fluctuations $[10,15,17]$ in chaotic systems.

The efficiency of this experimental approach, based on the prehistory distribution, was demonstrated earlier for noise-induced escape from a non-hyperbolic [18] chaotic attractor in a non-autonomous nonlinear oscillator $[10,19]$. Here we investigate a noise-induced escape from a quasi-hyperbolic [18] chaotic attractor in the Lorenz system, and thus address and answer the question: how does the mechanism of escape from a chaotic attractor depend on the structure of the attractor? In doing so, we confirm the applicability of our experimental approach [12-14] to a wider class of complex systems and we demonstrate that it provides the insight necessary for the application of analytical methods.

\section{The Model and Results}

The Lorenz system $[20,21]$ is of interest because it describes e.g. convective fluid dynamics, as well as the single mode laser [1,22]:

$$
\begin{aligned}
\dot{q}_{1}=\sigma\left(q_{2}-q_{1}\right), \quad \dot{q}_{2} & =r q_{1}-q_{2}-q_{1} q_{3}, \quad \dot{q}_{3}=q_{1} q_{2}-b q_{3}+\xi(t) \\
\langle\xi(t)\rangle & =0, \quad\langle\xi(t) \xi(0)\rangle=D \delta(t) ;
\end{aligned}
$$

here $q_{1}, q_{2}, q_{3}$ are dynamical variables, and $\sigma, r, b$ are parameters. For simplicity let us consider the noise source to act through the third equation only. This does not destroy the mirror symmetry of the system, which is invariant under the change of variables $\left(q_{1}, q_{2}, q_{3}\right) \rightarrow\left(-q_{1},-q_{2}, q_{3}\right)$. The situation when noise affects all three variables is more complicated for the analysis. First of all the dimension of the extended phase space is increased substantially and secondly adding noise to the first two equations lifts degeneracy of the optimal escape path. This situation, however, may be of importance in the context of analysis of the laser dynamics and will be considered elsewhere. 
For $\sigma=10, b=8 / 3, r=24.08$, the system (1) has three attractors [21]: two stable points $P_{1}$ and $P_{2}$, and the Lorenz attractor (Fig. 1(a)). Stable manifolds of the saddle cycles $L_{1}$ and $L_{2}$ surround the stable points and they constitute boundaries between the chaotic and regular regimes in this region of phase space. The chaotic Lorenz attractor consists of non-trivial hyperbolic sets, the separatrixes $\Gamma_{1}$ and $\Gamma_{2}$ and the saddle point $O$ [23].

The last fact can be illustrated (Fig. 1(b)) by the existence of two trajectories $T_{1}$ and $T_{2}$ (found by variation of the initial conditions on the attractor). These trajectories lie in the very close $\left(\propto 10^{-6}\right)$ vicinity of the stable manifold $W_{s}$ of the saddle point $O$. Accordingly they follow this manifold to pass nearby the point $O$. After that they approach to the saddle cycles $L_{1}$ and $L_{2}$ following the separatrixes $\Gamma_{1}$ and $\Gamma_{2}$ that define the boundary of the chaotic attractor [23]. Numerical studies have shown that the probability of the noise-free system passing near the separatrixes or the cycles $L_{1}$ and $L_{2}$ is exponentially small. In fact, in the noise-free system no trajectories can be found approaching a close vicinity of the cycles $L_{1}$ and $L_{2}$ along the separatrixes on any reasonable timescale of observation (a few weeks in our case).

However, by adding a very weak noise to the system $(D=0.001)$ one can induce transitions to the states $P_{1}$ and $P_{2}$, so that the Lorenz attractor becomes metastable.

To investigate noise-induced escape from the Lorenz attractor we have carried out numerical simulations implementing a high-speed pseudo-random generator [24] for the increment $\Delta q_{3}$. We build the prehistory distributions both for the escape trajectories and for the corresponding realizations of random force. For definiteness, we examine escape to the stable point $P_{1}$.
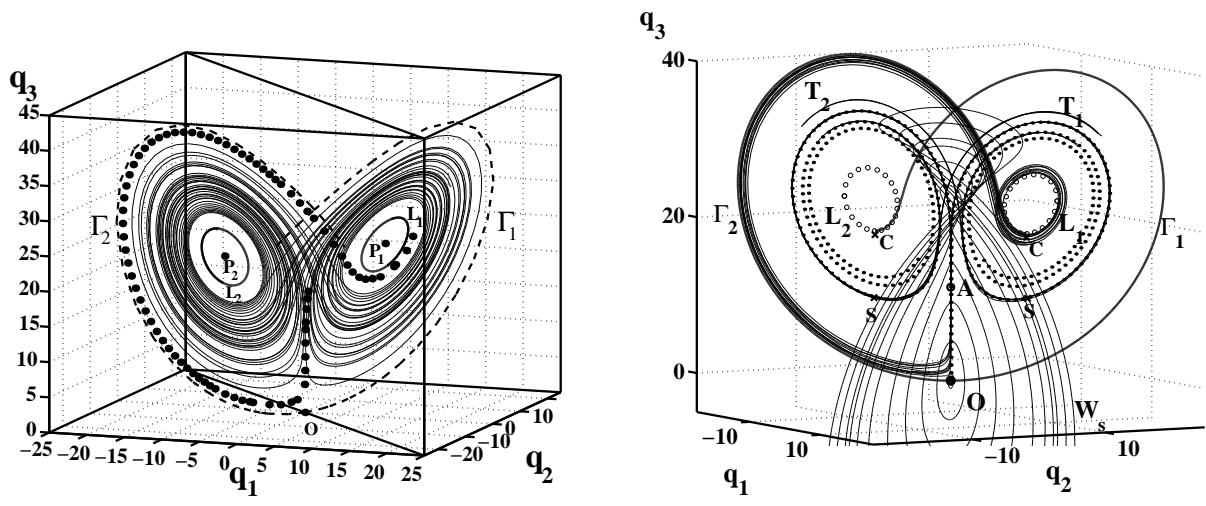

Fig 1. (a) The structure of phase space of the Lorenz system. A trajectory of the Lorenz attractor is shown by thin solid line, the separatrixes $\Gamma_{1}$ and $\Gamma_{2}$ are shown by dashed lines, one of the escape trajectories is shown by points. (b) 10 escape trajectories obtained by numerical simulation of (1) are shown by the grey lines. The trajectories $T_{1}$ and $T_{2}$ are shown by points, the cycles $L_{1}$ and $L_{2}$ are shown by circles. The stable manifold $W_{s}$ of the saddle point $O$ is shown by the fan of thin solid lines.

Ten escape trajectories obtained by numerical simulations are shown in Fig. 1(b). They approach the saddle point $O$ closely in two narrow bunches that emerge from the attractor. The tube shown is slightly dispersed near point $O$ and, moving along 


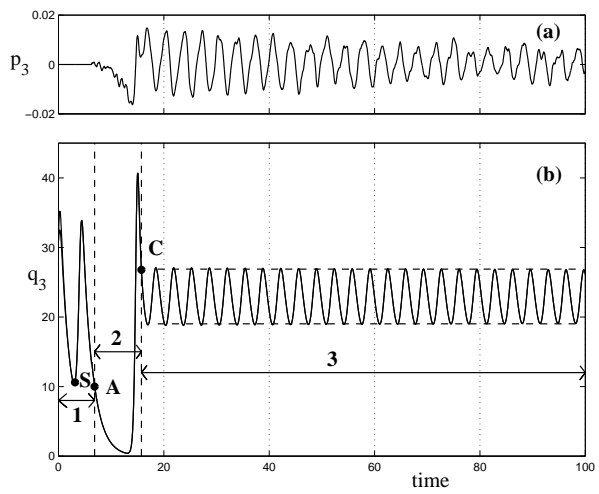

Fig 2. (a) The averaged fluctuational force $p_{3}$ after low-pass filtration and (b) the coordinate $q_{3}$ of optimal escape trajectory are shown. The three stages of escape are indicated by the numbers 1-3 by the corresponding double-headed arrows.

the separatrix $\Gamma_{2}$, it falls onto the unstable manifold of the saddle cycle $L_{1}$ in the vicinity of point $C$. The existence of two bunches of trajectories, leading from the points $S$ to the point $A$, is a consequence of the symmetry of the Lorenz system: it allows us to consider only one of these bunches leading to the point $A$, without loss of generality. Note that there are several ways to approach point $S$ within a chaotic attractor.

Analysis of the prehistory distribution of the escape trajectories shows that there is well-defined maximum in their distribution. Therefore we can define the optimal path by ensemble-averaging the escape trajectories. The optimal fluctuational force can be obtained by averaging the corresponding realizations of the random force. Fig. 2 shows the coordinate $q_{3}$ of the optimal path and the value $p_{3}$ of the averaged fluctuational force as functions of time during the escape process.

The results obtained suggest the following scenario of escape from the Lorenz attractor. The escape path consists of three main parts (Fig. 2(b)): (i) the deterministic part from the point $S$ to the point $A$; (ii) a part corresponding to fast fluctuation-assisted motion along the separatrixes from the point $A$ to the point $C$; and (iii) slow diffusion from the point $C$ to overcome the deterministic drift on the unstable manifold of the saddle cycle $L_{1}$ and to cross $L_{1}$. It is important to note that noise plays a different role during each part of the escape.

In the first part (from the point $S$ to the point $A$ ) the trajectory moves on the chaotic attractor. This path can be observed in the absence of noise, and the presence of noise results merely in a small diffusion of trajectories around the escape path. The fluctuational force (Fig. 2(a)) is equal to zero in this part. The probability $\rho_{1}$ to find the system in a small (of order $\sqrt{D}$ ) region near point $A$ is practically independent of the presence of noise, and it defines the frequency of the escape attempts. The independence of $\rho_{1}$ on noise intensity confirms the wellknown result $[3,25]$ that the structure of the Lorenz attractor does not change in the presence of fluctuations.

The second part corresponds to motion along the manifolds of the saddle point $O$ : (i) the stable two-dimensional manifold $W_{s}$ and (ii) the one-dimensional unstable 
manifold $\Gamma_{2}$. The distribution of escape trajectories has a finite width near the point $A$. If the noise is switched off at this point, then the trajectories will diverge from the manifolds $W_{s}$ and $\Gamma_{1}$ and, consequently, from the escape path. Under the action of fluctuations the trajectories remain in the vicinity of the manifolds and fall onto unstable manifolds of the saddle cycle $L_{1}$ near the point $C$. The fluctuational force corresponding to motion from point $A$ to point $C$ is different from zero and has a well defined shape (Fig. 2(a)). The time variation of the fluctuational force during this part of the escape trajectory is strongly correlated with the time dependence of coordinate $q_{3}$, and the action of the fluctuational force is always such that it pushes the system towards the separatrix. Note that the second part of the escape path can be observed, at least in principle, for the noise-free system if the system trajectory falls to within a very small vicinity (of order $10^{-6}$ ) of the trajectories $T_{1}$ or $T_{2}$. As pointed out above, however, the probability of such events occurring in practice is effectively zero, and none were observed on the few-weeks time scale of our experiments.

The third and final part of the escape trajectory corresponds to motion on the two-dimensional unstable manifold of the saddle cycle $L_{1}$. The escape trajectories fall to the unstable manifold, and then cross the cycle $L_{1}$ under the action of fluctuations. After crossing, the trajectories relax to the stable point $P_{1}$. In the absence of fluctuations the trajectory will spiral very slowly inwards from the saddle cycle $L_{1}$. The velocity of slow (averaged over a period) drift is defined by the largest multiplier of the saddle cycle $L_{1}$, which is equal to $\mu_{1}=1.028$. Because the deterministic drift of the system away from the saddle cycle $L_{1}$ is very slow, it can be overcome by the diffusion of the system across the cycle. However, only those components of the noise that oscillate in anti-phase with the coordinate $q_{3}$ can contribute to the crossing of the limit cycle (recall that the noise acts only on the $q_{3}$ component of our system, so that it is only the oscillations of the fluctuational force in anti-phase with $q_{3}$ that can push the system towards the limit cycle). This fact is reflected in the long anti-phase oscillations of the fluctuational force in Fig. 2(a). Obviously, this part of the escape processes relies entirely on the action of noise.

The long time interval over which the third part of the escape process occurs suggests that the coupling between this part and the two earlier parts is only probabilistic, and that there is no dynamical coupling between them.

\section{Conclusion}

We conclude that, for a quasi-hyperbolic attractor, there exists a unique optimal escape path. The role of fluctuations is two-fold. First, they deliver the system to a seldom-visited region in the neighborhood of the saddle cycle $L_{1}$. And secondly they induce the system to cross the saddle cycle $L_{1}$. The escape path consists of three parts (Fig. 2(b)). The motion during the first part is deterministic, but it is governed by a non-zero fluctuational force during the second and third parts of the escape process. Therefore the escape probability can at least in principle be found as a product of probabilities of sequential transitions $\rho_{1}, \rho_{2}$ and $\rho_{3}$. Here $\rho_{1}$ is the probability to find the system in some specific region in the vicinity of trajectories $T_{1}$ or $T_{2}$ in the absence of noise, $\rho_{2}$ is the probability of a transition from the point $A$ to a given region on the two-dimensional manifold of the saddle cycle $L_{1}$, and $\rho_{3}$ 
is the probability of crossing the saddle cycle $L_{1}$.

The probability $\rho_{1}$ can be found as a probability measure of the Lorenz attractor in the absence of noise. $\rho_{2}$ is defined by the dynamics of the system in the deterministic flow near the separatrix in the presence of fluctuations. The motion takes place during a finite time interval, and the role of fluctuations is to push the system as close as possible towards the separatrix. The probability $\rho_{3}$ is determined by the stochastic dynamics of the system on the two-dimensional unstable manifold of the saddle cycle. The analysis of $\rho_{3}$ can be reduced to an analysis of the stochastic dynamics of the slowly varying amplitude of oscillations near $L_{1}$. In this approximation one has to find the probability of a Brownian particle crossing a potential barrier that is set initially in the close vicinity of the potential maximum.

The theoretical analysis of the probabilities $\rho_{2}$ and $\rho_{3}$ will be considered later in a paper to be published elsewhere.

Finally, we emphasize that our observations of a unique optimal escape path and the corresponding optimal fluctuational force, based on experimental measurements of the prehistory probability distribution [12], pave the way for further theoretical analysis of the stability of the Lorenz attractor in the presence of fluctuations, and of fluctuational dynamics within a chaotic attractor.

\section{Acknowledgements}

We are very grateful to M.I. Dykman and V.N. Smelyanskiy for their continuing interest in our work and many valuable discussions of results. The research was supported by the Engineering and Physical Sciences Research Council (UK) and by Award No. REC-006 of the U.S. Civilian Research \& Development Foundation for the Independent States of the Former Soviet Union (CRDF).

\section{References}

\section{References}

[1] R. Graham, Macroscopic potentials, bifurcation and noise in dissipative systems, in Noise in Nonlinear Dynamical Systems vol. 1, eds. F. Moss and P.V.E. McClintock, Cambridge University Press, Cambridge (1989) 225-278.

[2] V.S. Anishchenko, A.B. Neiman. Structure and properties of chaos in presence of noise, Nonlinear Dynamics of Structures, eds. R.Z. Sagdeev, U. Frich, S.S. Moiseev and N. Ekorkin, World Scientific, Singapore (1991) 21-48.

[3] R. Graham, A. Hamm and T. Tel, Nonequilibrium potentials for dynamical systems with fractal attractors or repellers, Phys. Rev. Lett. 66 (1991) 3089-3092.

[4] R.L. Kautz, Noise, chaos, and the Josephson standard, Rep. Prog. Phys. 59 (1996) 935-992.

[5] Nonlinear Dynamics and Spatial Complexity in Optical Systems, eds. R.G. Harrison, J.S. Uppal and P. Osborne (SUSSP/Institute of Physics, Bristol, 1993); E. Arimondo, D. Hennequin, P. Glorieux, Noisy dynamics in optically bistable systems, in Noise in Nonlinear Dynamical Systems vol. 3, eds. F. Moss and P.V.E. McClintock, Cambridge University Press, Cambridge (1989) 119-158.

[6] P. Faure and H. Korn, A nonrandom dynamic component in the synaptic noise of a central neuron, Proc. Natl. Acad. Sci. USA 94 (1997) 6506-6511; H.A. Braun, K. Schafer, K. Voigt, R. Peters, F. Bretschneider, X. Pei, L. Wilkens, F. Moss, Low- 
dimensional dynamics in sensory biology .1. Thermally sensitive electroreceptors of the catfish, J. Comp. Neursc. 4 (1997) 335-347.

[7] R.D. Astumian and I. Derenyi, Fluctuation driven transport and models of molecular motors and pumps, Eur. Biophys. J. with Biophys. Lett. 27 (1998) 474-489.

[8] E.A. Jackson, The OPLC control methods for entrainment, model-resonance, and migration actions on multiple-attractor systems, Chaos 7 (1997) 550-559.

[9] B. Hubinger, R. Doerner, W. Martienssen, M. Herdering, R. Pitka, and U. Dressler Controlling chaos experimentally in systems exhibiting large effective Lyapunov exponents, Phys. Rev. E 50 (1994) 932-948.

[10] I.A. Khovanov, D.G. Luchinsky, R. Mannella and P.V.E. McClintock, Fluctuations and the energy-optimal control of chaos, Phys. Rev. Lett. 85 (2000) 2100-2103.

[11] M.I. Freidlin and A.D. Wentzel, Random Perturbations in Dynamical Systems, Springer, New-York (1984).

[12] M.I. Dykman, P.V.E. McClintock, V.N. Smelyanski, N.D. Stein and N.G. Stocks, Optimal paths and the prehistory problem for large fluctuations in noise-driven systems, Phys. Rev. Lett. 68 (1992) 2718-2721.

[13] M.I. Dykman, D.G. Luchinsky, P.V.E. McClintock, and V.N. Smelyanskiy, Corrals and critical behavior of the distribution of fluctuational paths, Phys. Rev. Lett. 77 (1996) 5229-5232.

[14] D.G. Luchinsky and P.V.E. McClintock, Irreversibility of classical fluctuations studied in analogue electrical circuits, Nature 389 (1997) 463-466.

[15] D.G. Luchinsky, P.V.E. McClintock and M.I. Dykman, Analogue studies of nonlinear systems, Rep. Prog. Phys. 61 (1998) 889-997.

[16] L. Onsager and S. Machlup, Fluctuations and irreversible processes, Phys. Rev. 91 (1953) 1505-1512.

[17] V.N. Smelyanskiy and M.I. Dykman, Optimal control of large fluctuations, Phys. Rev. E 55 (1997) 2516-2521.

[18] V. Anishchenko and G. Strelkova, Irregular attractors, Discrete Dynamics in Nature and Society 2 (1998) 53-72.

[19] D.G. Luchinskiy and I.A. Khovanov Fluctuation-induced escape from the basin of attraction of a quasiattractor, JETP Lett. 69 (1999) 825-830.

[20] E.N. Lorenz Deterministic nonperiodic flow, J. Atmos. Sci. 20 (1963) 130-141.

[21] C. Sparrow, Lorenz Equations: Bifurcations, Chaos and Strange Attractors, Applied Mathematical Sciences vol.41, Springer-Verlag, New-York (1982).

[22] H. Haken, Analogy between higher instabilities in fluids and lasers, Phys. Lett. A 53 (1975) 77-78.

[23] V.S. Afraimovich, V.V. Bykov and L.P. Shilnikov, On attracting structurally unstable limit sets of Lorenz attractor type (in Russian), Tr.y Mos. Mat. Obs. 44 (1982) 150212.

[24] G. Marsaglia and W.-W. Tsang, A fast, easily implemented method for sampling from decreasing or symmetric unimodal density-functions, SIAM J. Sci. Stat. Comput. 5 (1984) 349-359.

[25] Ya.I. Kifer, On small random perturbations of diffeomorphisms, Uspekhi matematicheskikh nauk 29 (1974) 173-174.

[26] R.S. Maier and D.L. Stein, Limiting exit location distributions in the stochastic exit problem, SIAM J. Appl. Math. 57 (1997) 752-790.

[27] V.N. Smelyanskiy, M.I. Dykman and R.S. Maier, Topological features of large fluctuations to the interior of a limit cycle, Phys. Rev. E 55 (1997) 2369-2391. 